\title{
Examining traffic flow parameters at merging section on high-speed urban roads in India
}

\author{
Pallav Kumar ${ }^{1, *}$, Shriniwas Arkatkar ${ }^{2}$ and Gaurang Joshi ${ }^{2}$ \\ ${ }^{1}$ Civil Engineering Department, Muzaffarpur Institute of Technology, Muzaffarpur 842 003, India \\ ${ }^{2}$ Civil Engineering Department, Sardar Vallabhbhai National Institute of Technology, Surat 395 007, India
}

\begin{abstract}
This study analyses micro-level and macro-level traffic parameters at merging location on high-speed urban roads in India. For this purpose, representative merging section was selected on Delhi-Gurgaon expressway. Speeds on different lanes at merging section were found to gradually decrease from median side lane to lane closer to merging point. At low and moderate flow conditions, most of the vehicles avoid moving in lanes closer to merging point in the main traffic stream. This is done to reduce the crash as well as to avoid competing for the same road space. Speed versus flow relationship developed for basic roadway section and merging section shows the difference in capacity by almost $8 \%$ due to merging operation. Moreover, stream speed at the merging is found to be the function of main stream flow and entry flow. The findings from the study would be useful in operational analysis of merging sections on high-speed urban roads in India.
\end{abstract}

Keywords: Heterogeneous traffic, merging section, time headway, speed distribution, speed-flow relationship.

HiGHSPEED urban roads are one of the highest categories of roads in India connecting urban areas with divided carriageway for high-speed travel having partial control of access at frequent locations such as basic, weaving, merge and diverge segments. Contrary to the expressways, the movement of smaller vehicles such as motorized two-wheelers and motorized three-wheelers is normally permitted. For capacity and level of service analysis on this type of facility, extended lengths of roadway composed of continuously connected basic, weaving, merge and diverge segments should be considered separately. Exit and entry ramps are essential components of such roadway facilities to provide access or egress at designated locations, where traffic merges or diverges. To evaluate the status of traffic flow and its consequent effect on capacity and safety of operation of the facility, at the location of merging, it is necessary to study variation in traffic parameters like flow, individual vehicle speed and time headway at the location of merging section. This study is conducted to examine the traffic

\footnotetext{
*For correspondence. (e-mail: pallav318@gmail.com)
}

flow characteristics at merging location on high-speed urban roads having eight-lane divided carriageway.

Among the expressway segments, merging sections are usually regarded as the most important and complicated ones. Several studies have been conducted to study the driver behaviour at the location of merging sections ${ }^{1-3}$. These are potential locations where the breakdown in operation occurs. The reason is mainly due to two traffic streams competing for the same road space ${ }^{4}$.

Sarvi and Kuwahara ${ }^{5}$ studied the impact of heavy vehicles travelling on the expressway upon merging section capacity and concluded that increasing 1 percentage of heavy vehicles results in $1 \%$ decrease in capacity. Sarvi et al. ${ }^{6}$ utilized traffic simulation approach to test the proposal of the lane closure. They found that closures of on-ramp lane or closure of both on-ramp lane and mainline lane increase capacity by almost $3 \%$. They also used a simulation model to investigate the benefit of deploying VMS before the merging point to guide truck drivers to switch lanes to avoid conflicts with merging vehicles. They concluded that by moving $10 \%$ of heavy vehicles, the capacity of the merging section could be improved by $1 \%$. Merging sections are one of the main sources of traffic crashes on expressways ${ }^{7}$. It is quite evident from literature review that competition of merging and mainline flows for the same capacity at downstream of the merging point can be major causes of breakdown and capacity drop. To reduce the effect of merging vehicles on the mainline traffic, several control strategies have been applied so that the capacity of merging sections can be improved. The control strategies are carried out by managing the mainline traffic flow and/or merging traffic flow. However, intelligent transportation system (ITS)based approaches such as variable message sign (VSM) or ramp metering can be applied. Moreover, traffic control strategies can also be applied by changing the physical layouts of merging sections such as lane closure, changing the length of chevron marking or the length of the acceleration lane.

\section{Objectives}

Aashto $^{8}$ stated that the acceleration lanes of merging sections should have sufficient length to enable a driver to make appropriate change in speed between the merging 
and mainline vehicles. But in the traffic conditions prevailing on high-speed urban corridors in India, the merging location is inadequately designed with the smaller length of acceleration lanes. This kind of merging manoeuver is also quite common on national highways in India. Moreover, there is hardly any study in India pertaining to merging manoeuver on such high-speed urban roads. The present study considers the traffic flow operations on such merging locations on high-speed urban roads in India. The primary objectives are:

- To study the effect of merging on speeds of vehicles across different lanes at the merging section.

- To check the effect on free speed distribution of different vehicle categories due to merging location.

- To analyse the effect on time headway at various locations (at upstream of merging, at the location of merging and basic section, which is $1 \mathrm{~km}$ away from the merging location) and at different flow levels.

- To study the macroscopic speed-flow relationship at the merging location.

- To study the effect of entry flow and mainstream flow and its stream speed at the location of merging.

\section{Data collection}

For field data collection, a merging section was selected on an eight-lane divided multilane urban road in Delhi. The selected location can be considered as a representative section, based on reconnaissance survey conducted over the entire roadway stretch of $28 \mathrm{~km}$. Several such merging sections were visited before making the final decision. The location was selected such that a camera can be fixed at a vantage point to capture the traffic operation taking place at the merging location. The plan of the study section given in Figure $1 a$ shows that the overall road segment considered with four-lanes in one-direction can be clearly visualized.

(a) Upstream of merging location: It is a point lying at $30 \mathrm{~m}$ upstream of a section at merging. At this location, time headway is only studied to understand the behaviour of vehicles prior to merging location.

(b) Section at merging: It is a $65 \mathrm{~m}$ long section just at the verge of merging. It is the location where traffic flow parameters such as speed, flow rate and headways are studied.

(c) Basic section: To compare the effect of merging on traffic flow parameters, traffic data is also collected at the location $1 \mathrm{~km}$ away from the location of the merging section. This section does not possess any kind of influence on its uninterrupted flow.

The google map of the study location is shown in Figure $1 \mathrm{~b}$. The roadway section is eight-lane divided urban road having a carriageway width of $14 \mathrm{~m}(3.5 \mathrm{~m} \times 4$ lanes $)$ on each side. Traffic video was captured by placing three cameras. The first one at upstream of merging section (as shown in Figure $1 c$ ) and another focused towards section at merging (as shown in Figure $1 d$ ). The third camera was placed at the location of basic section which is $1 \mathrm{~km}$ away from the merging location (as shown in Figure $1 e$ ). Traffic video was collected for the duration of $6 \mathrm{~h}$ from $6 \mathrm{am}$ to $12 \mathrm{pm}$ using three coordinated cameras on 17 March 2017 with three coordinated cameras. The duration was so selected to capture both non-peak hour and peak hour traffic flow.

\section{Data extraction}

The recorded traffic video was played on the computer screen using Avidemux 2.6 software. Using the field geometric details, grid lines were prepared in AutoCAD software for the study stretch and the same was overlaid on the recorded video which was played in Avidemux to make data extraction procedure convenient. To start the extraction work, traffic volume for 1 min interval was done manually by counting the vehicles passing through the section. The manually counted volume was simultaneously entered in Microsoft Excel sheet for further analysis. Moreover, the time stamp at entry and exit of a vehicle through the upstream and downstream of the section was also noted to get time headway and speed data for further analysis process. The total duration of $6 \mathrm{~h}$ of traffic flow data was extracted from the traffic video of $6 \mathrm{~h}(6: 00 \mathrm{am}$ to $12: 00 \mathrm{pm})$. The duration was selected so that maximum variation in traffic flow was captured based on visual inspection.

\section{Observations on traffic flow}

\section{Overall traffic composition}

The traffic composition analysed over the study section for the entire duration is given in Figure 2. The study section has the highest proportion of car $(81 \%)$ as shown in Figure 2. It is followed by motorized two-wheeler (2W) composition, which is around $14 \%$ in the traffic stream and lower proportion of heavy vehicle in the traffic stream $(1 \%$ - bus, $1 \%$ - truck) was observed. Motorized three-wheeler $(3 \mathrm{~W})$ and $\mathrm{LCV}$ was observed to be around $2 \%$ and $1 \%$ respectively.

\section{Flow variation at merging location}

The variation in traffic flow over the study section is shown in Figure 3. The mainstream flow for the observed duration lied between 2000 vehicles/hour/direction and 12,000 vehicles/hour/direction for eight-lane divided 

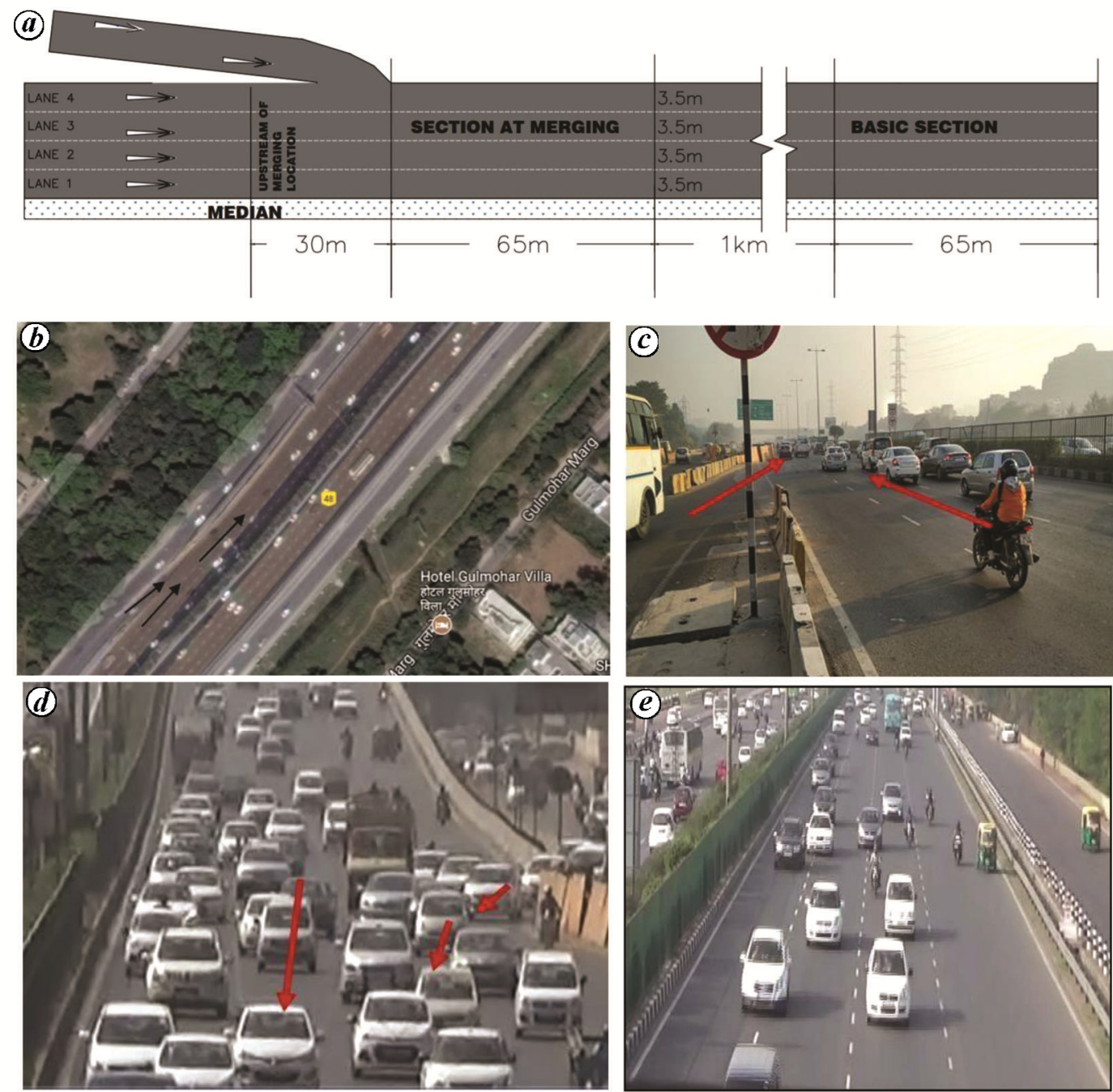

Figure 1. Study section details. $\boldsymbol{a}$, Overall plan of study section. $\boldsymbol{b}$, Google map of the merging section located on DelhiAjmer expressway. Section at merging from: $(\boldsymbol{c})$ camera 1 and $(\boldsymbol{d})$ camera $2 . \boldsymbol{e}$, Basic section which is $1 \mathrm{~km}$ away from merging section.

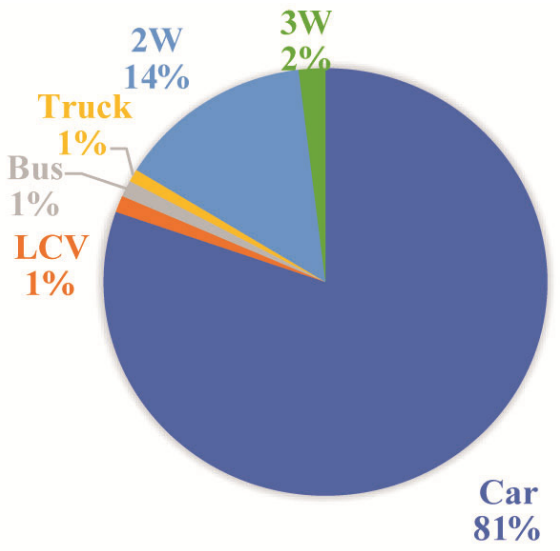

Figure 2. Traffic composition on study section.

study section. It was observed that the range of flow merging into the main traffic stream lied between 300 and 3000 vehicles/hour. The pattern of flow variation in the main traffic stream and entry flow can be very well observed through Figure 3. For analysis on traffic flow, Figure 3 shows distribution of traffic on roadway sections 6:00 am to $12: 00 \mathrm{pm}$ at interval of $1 \mathrm{~min}$. The reason for selecting $1 \mathrm{~min}$ interval is to show the maximum variation in observed traffic over the roadway section.

\section{Lane-wise traffic composition}

On high-speed urban roads in India, it is common that the fast-moving vehicles like car move in the lane closer to the median side whereas slow moving vehicles are mostly restricted towards kerbside lanes. However, this behaviour is somewhat uncommon in the case of merging section, lanes which are closer to kerbside also have a significant proportion of vehicles from all categories because of the entry flow location close to the kerbside lanes. This behaviour was studies to show the variation in 


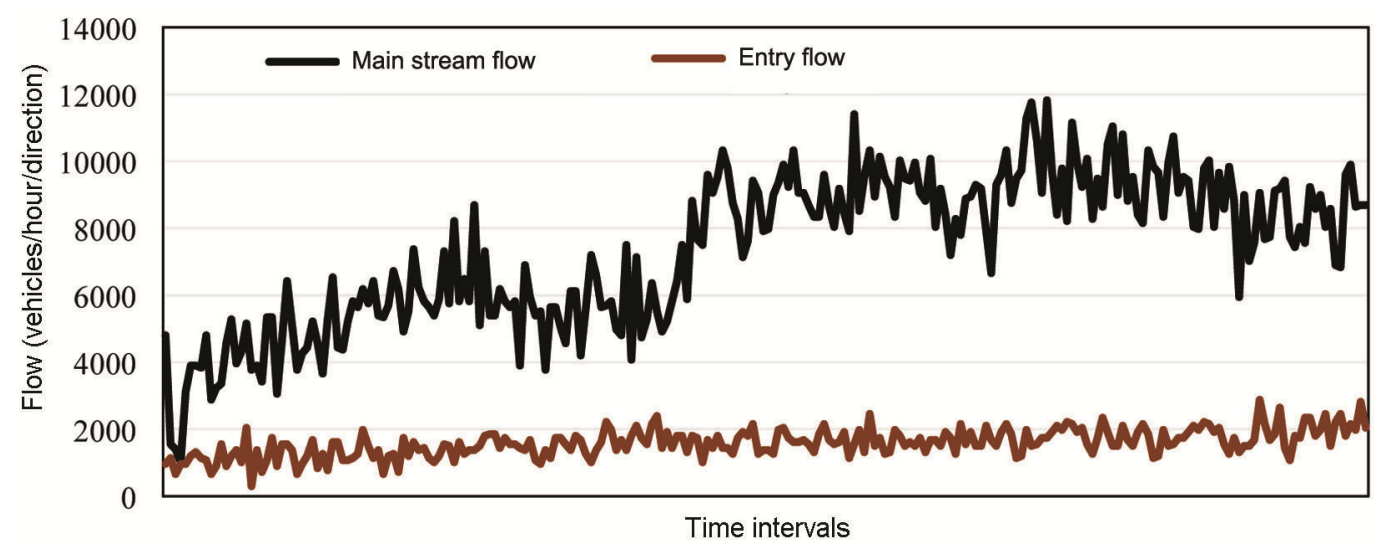

Figure 3. Mainstream flow and entry flow at the location of merging.

Table 1. Percentage vehicle composition for different lanes at merging section

\begin{tabular}{|c|c|c|c|c|c|c|}
\hline Vehicle category & $2 \mathrm{~W}$ & $3 \mathrm{~W}$ & Car & Bus & LCV & Truck \\
\hline \multicolumn{7}{|c|}{ Low flow conditions $(V / C$ ratio $<0.4)$} \\
\hline Lane $1(\mathrm{~L} 1)$ & & - & 97 & 1 & - & 1 \\
\hline Lane 2 (L2) & 2 & - & 94 & 1 & & - \\
\hline Lane 3 (L3) & 6 & 1 & 86 & 4 & 1 & 1 \\
\hline Lane 4 (L4) & 42 & 4 & 47 & 2 & 1 & 2 \\
\hline \multicolumn{7}{|c|}{ Moderate flow conditions $(V / C$ ratio 0.4 to 0.7 ) } \\
\hline Lane 1 (L1) & - & - & 100 & - & - & - \\
\hline Lane 2 (L2) & 1 & - & 98 & - & 1 & - \\
\hline Lane 3 (L3) & 14 & 1 & 80 & 3 & - & 1 \\
\hline Lane 4 (L4) & 48 & 3 & 42 & 2 & 2 & 1 \\
\hline \multicolumn{7}{|c|}{ Heavy flow conditions ( $V / C$ ratio 0.7 to 1.0 ) } \\
\hline Lane $1(\mathrm{~L} 1)$ & - & - & 99 & - & - & - \\
\hline Lane 2 (L2) & 3 & - & 95 & 1 & 3 & - \\
\hline Lane 3 (L3) & 19 & 2 & 75 & 1 & 2 & 1 \\
\hline Lane 4 (L4) & 52 & 1 & 38 & 2 & 1 & 1 \\
\hline
\end{tabular}

traffic composition for all the four lanes in the study section and is presented in Table 1. The variation in traffic composition was studied at different flow levels across different lanes. The flow conditions were divided into three parts: (a) Low flow conditions (when volumeto-capacity $(V / C)$ ratio $<0.4)$. (b) Moderate flow conditions (when $V / C$ ratio is between 0.4 and 0.7 ). (c) Heavy flow conditions (when $V / C$ ratio is between 0.7 and 1.0). The $V / C$ ratio taken here is based on the speed-flow curve in Figure 7. Based on the speed-flow relationship developed in Figure 7, the capacity of the study section is achieved by calibration of constant parameters in Northwestern model, discussed later in this study. Further, the flow value of $5 \mathrm{~min}$ intervals was divided by capacity, which gives $V / C$ ratio for each 5 min intervals. Later, the $V / C$ ratio data were sorted based on increasing order. Then, the analysis of traffic composition, time headway and speed was made for different flow-levels. The lane numbering pattern in this study can be clearly understood from Figure $1 a$.

Table 1 indicates that cars exceptionally dominate the first two lanes from median-side (lanes 1 and 2). It is a special case of homogeneity in traffic on high-speed urban roads in India. The last two lanes, lane 3 (adjacent to kerbside lane) and lane 4 (kerbside lane) have flow from almost all vehicles categories including cars, motorized two-wheelers, motorized three-wheelers, LCV and heavy vehicles (bus and truck). The proportion of different vehicle categories in lanes 3 and 4 is due to the location of a section at merging. The subsequent section presents the speed data for different lanes for the study section. Moreover, spot speed survey conducted at $1 \mathrm{~km}$ downstream (basic section) of and at the merging section is also presented here to probe into the effect of merging on speed behaviour of different vehicle categories.

\section{Comparison of speeds for base section and at merging section}

Speed distribution of vehicles is conducted during low flow conditions. The flow during the spot speed study was observed below 3000 vehicles/hour/direction for four lanes in one direction. To study the effect of presence of 

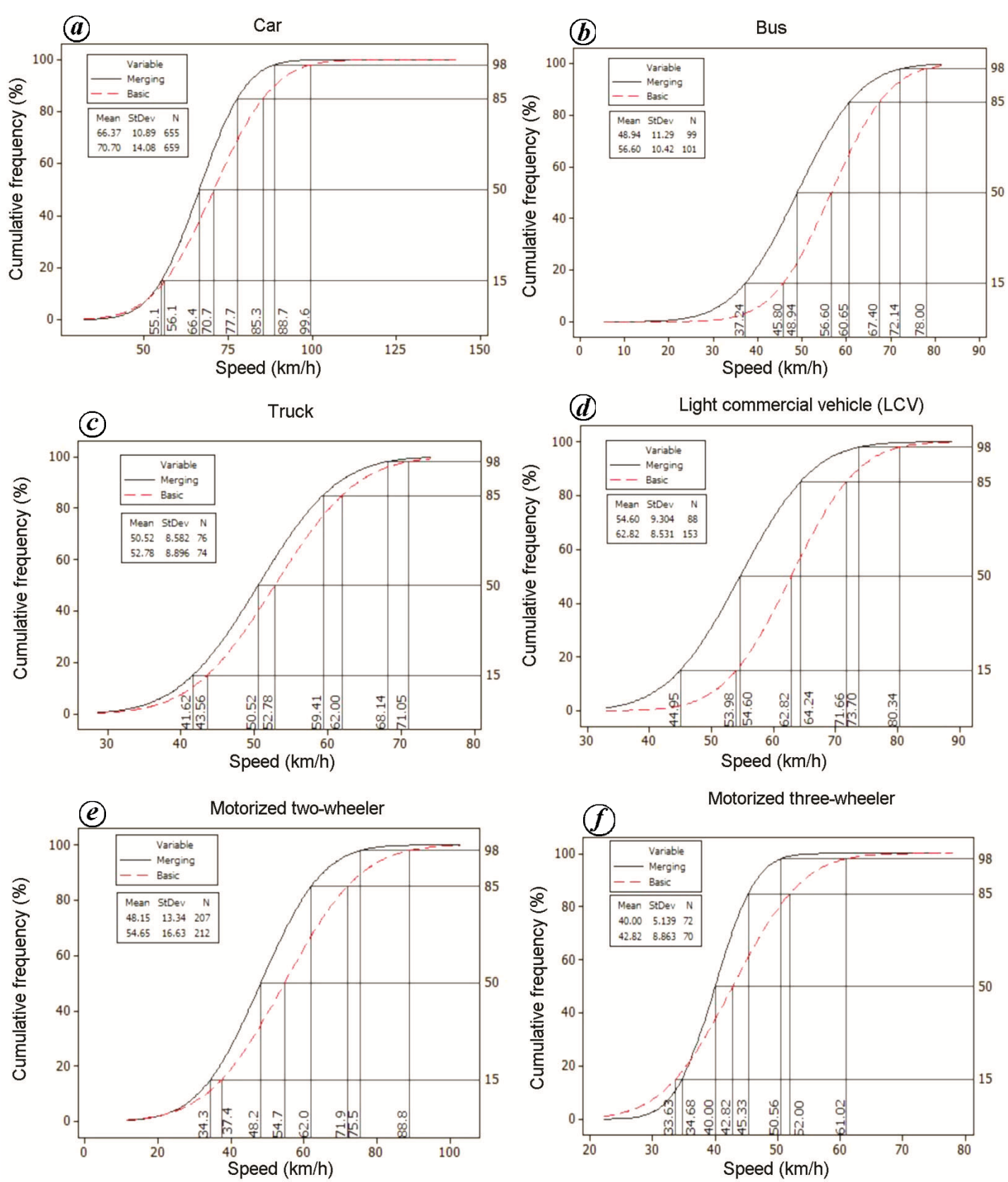

Figure 4. Free speed distribution at merging section and basic section for different vehicle categories: $\boldsymbol{a}$, Car; $\boldsymbol{b}$, Bus; $\boldsymbol{c}$, Truck; $\boldsymbol{d}, \mathrm{LCV} ; \boldsymbol{e}$, Motorized two-wheeler; $\boldsymbol{f}$, Motorized three-wheeler.

merging on speeds, spot speed study was carried out at the basic section (which is $1 \mathrm{~km}$ away from the study section) and merging section. The 15th, 50th, 85th and 98th percentile speeds are marked on the $Z$-axis of Figure 4. $X$-axis represents the speeds of the vehicle and $Y$-axis represents the percentage (\%) cumulative frequency.

From Figure 4, a significant reduction is seen in the speed of the vehicles due to the presence of a merging section on eight-lane divided high-speed urban roads. The mean percentage reduction in speed for different vehicle categories is in the range of $4 \%$ to $15 \%$ based on the mean of the observed speed data as shown in Table 2 .

\section{Lane-wise speed characteristics of vehicles at merging section}

Speed is a fundamental measurement of performance of a given roadway facility. The free flow speed measured at any section is a function of a number of factors such as road geometry, road surface conditions, vehicular characteristics, individual driver behaviour and other factors such as road-side environment, weather and time of the day. An understanding of the speed characteristics is of immense importance in traffic engineering. It indicates the quality of service experienced by road users. 
Table 2. Mean percentage reduction in speed of different vehicle categories

\begin{tabular}{lcccccc}
\hline Vehicle & Car & Bus & Truck & LCV & $2 \mathrm{~W}$ & $3 \mathrm{~W}$ \\
\hline Speed reduction (\%) & 5.7 & 14.2 & 4 & 12.9 & 11 & 4.7 \\
\hline
\end{tabular}
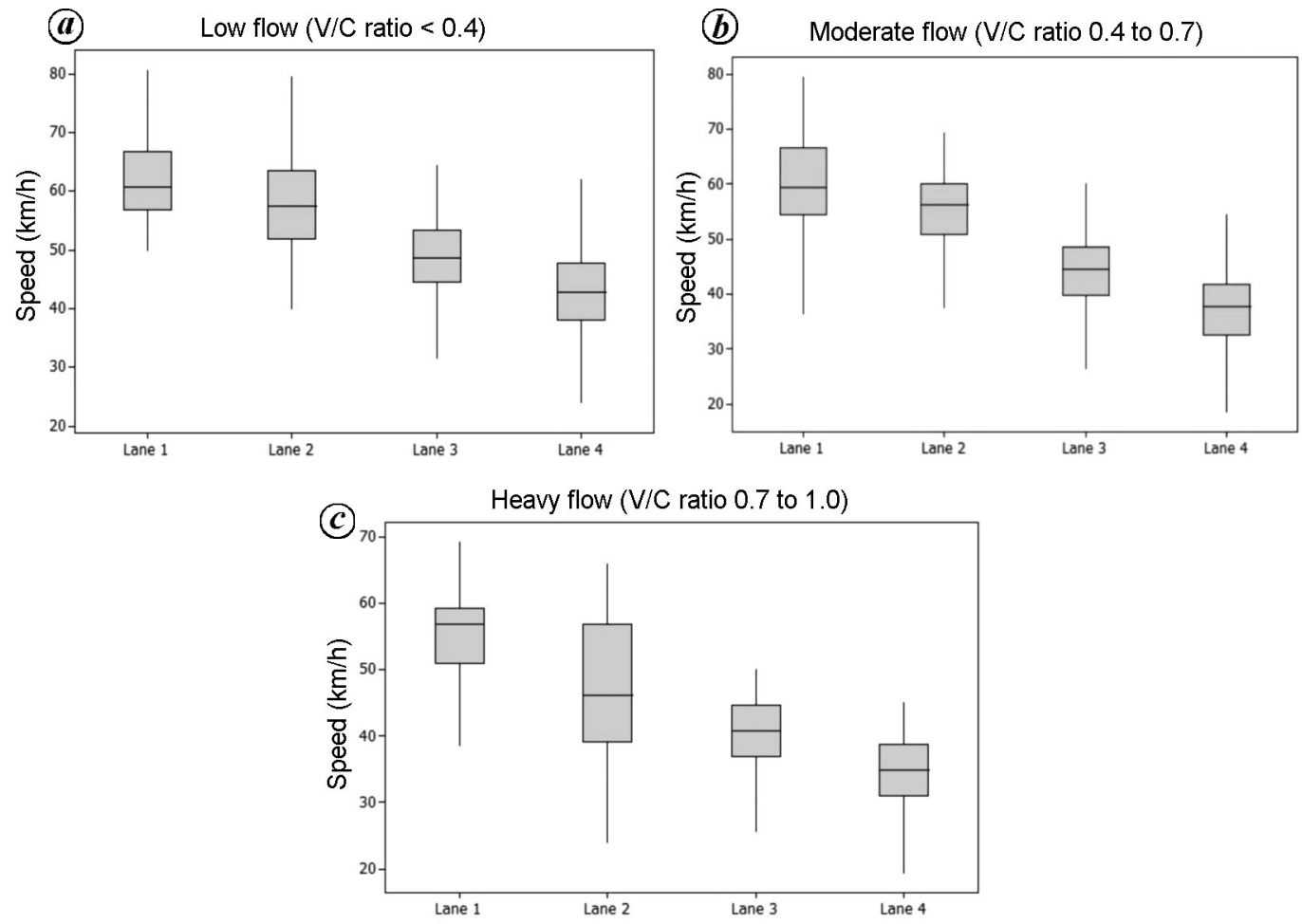

Figure 5. Speed distribution fitting for different lanes under: $\boldsymbol{a}$, low flow conditions; $\boldsymbol{b}$, moderate flow conditions; $c$, heavy flow conditions.

Moreover, it helps in further studies related to geometric design, traffic regulation and level of service. It was decided to explore the speed distribution pattern of the vehicles across different lanes at the merging section.

The box plot of speed-distribution was studied under three different flow conditions: (a) low flow conditions (when $V / C$ ratio $<0.4$ ); (b) moderate flow conditions (when $V / C$ ratio is between 0.4 and 0.7 ); (c) heavy flow conditions (when $V / C$ ratio is between 0.7 and 1.0); these are shown in Figure 5.

The speed distribution pattern across different lanes suggests a gradual reduction in the speed of vehicles across different lanes. Towards the median side lanes, vehicles move at higher speeds as against the kerbside lanes, where the vehicle speeds are found to be decreasing gradually. This is due to the maximum composition of cars towards former and higher heterogeneity in traffic towards latter. This observation was common for low flow moderate, flow and heavy flow conditions. Contrary to this, during heavy flow conditions, lane 2 has higher variation in speeds. It is attributed to the fact that during some instances vehicle in lane 2 moves at higher speed and in other cases, it gets equally influenced due to the merging manoeuver, which results in a heavy reduction in the speed of the vehicles. The influence on lane 2 due to merging manoeuver can be observed through Figure $1 d$.

\section{Effect of location parameters on headway of different lanes}

Statistical analysis of time headway is quite important for microscopic traffic parameter modelling. It describes an inherent pattern of the traffic flow. Such a statistical model is essential for empirical traffic modelling as well as in the calibration of simulation model. From the theoretical perspective, such a model will guide researchers to build up an analytical framework to describe traffic flow characteristics. For simulation models, these models will serve as the input based on its mathematical distribution. More specifically, a statistical distribution of time headways is required for generating a random temporal space between vehicles in a simulation. The headway analysis was conducted at various locations: (a) upstream of 


\section{RESEARCH ARTICLES}
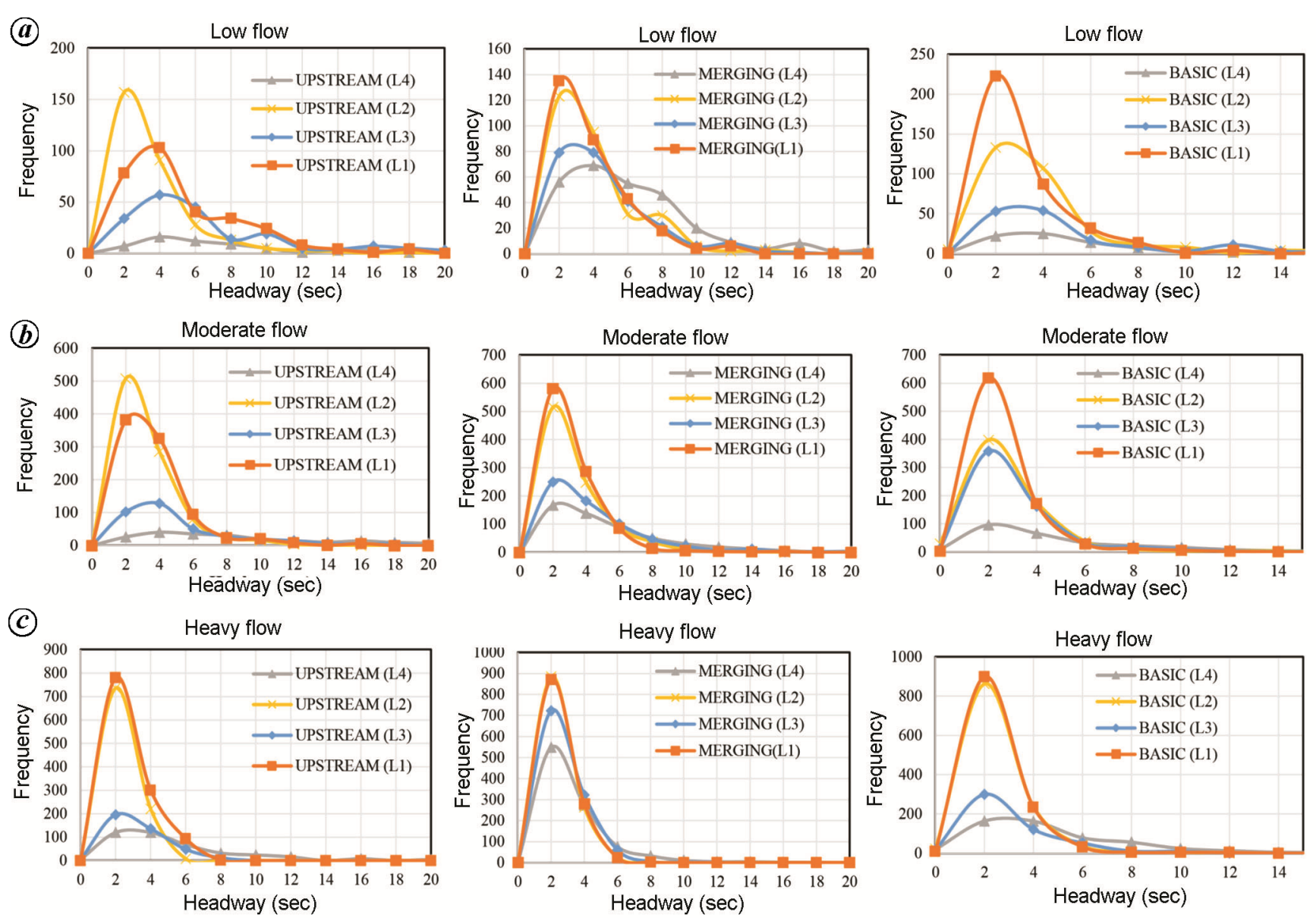

Figure 6. Lane-wise headway distribution under: $\boldsymbol{a}$, low flow at upstream, merging and basic sections; $\boldsymbol{b}$, moderate flow at upstream, merging and basic sections; $\boldsymbol{c}$, heavy flow at upstream, merging and basic sections (L1, Lane 1, L2, Lane 2, L3, Lane 3 and L4, Lane 4).

merging section; (b) at the location of merging; (c) basic study section, which is one $\mathrm{km}$ away from the merging section.

The headway analysis was first done to understand the pattern in which headway varied at various locations. Different distribution types were analysed such as gamma, exponential, negative exponential, shifted negative exponential, log-normal and generalized extreme value (GEV) distribution. Among them, GEV is found to fit well to the empirical dataset on headway across different lanes at 95\% confidence level and 5\% level of significance. The test was conducted using easy-fit software and resulted in GEV distribution being one of the best fit for headway data. Frequency distribution of headway for different lanes and at various locations (upstream, merging and basic section) for low flow $(V / C$ ratio $>0.4)$, moderate flow $(V / C$ ratio $0.4-0.7)$ and heavy flow conditions $(V / C$ ratio $0.7-1.0)$ are shown in Figure $6 a-c$ respectively. From Figure $6 a-c$ above, the following inferences can be drawn:

- Under low and moderate flow conditions, at upstream of the merging vehicles are more likely to be moving in lane 2 (L2) to avoid competing with merging vehicles for the same road space; vehicles from lane 3 and lane 4 shift towards lane 2. But the same is not possible under heavy flow conditions since a lesser degree of space is available to undergo lane-change manoeuvre for the vehicles.

- At the location of merging, all the lanes are utilized effectively under different flow conditions.

- Lanes 3 and 4 are least utilized at upstream and basic section; however, at the location of merging, more headway frequency can be seen for lane 2 . This is due to the presence of merging manoeuvre where vehicles are mainly found to be entering lanes 3 and 4; these lanes are also equally utilized by the vehicles.

- Under different flow conditions, at upstream, merging and basic section, vehicles are more likely to be moving in lanes 1 and 2. These are the lanes where vehicles are found to be moving at very high speed (clearly observed through Figure $5 a-c$ ) because these lanes are least affected due to merging manoeuvre under low and moderate flow conditions. However, under heavy flow conditions, lane 2 is also slightly affected that resulted in higher speed range (Figure $5 c$ ). 


\section{Speed-flow relationship at merging section and basic section}

Based on the empirical dataset, speed and flow data were aggregated at $5 \mathrm{~min}$ intervals for merging section and basic roadway section. Then, the macroscopic speed-flow relationship was developed at the merging section and basic section on eight-lane divided multilane urban road considered in the present study. To find out the effect of merging section on the capacity of multilane urban road in Delhi, a comparison was made on speed-flow relationship between basic section (which is $1 \mathrm{~km}$ downstream from the merging location) and merging section as shown in Figure 7. Flow in terms of PCU/hour/direction is obtained based on the methodology presented by Kumar et al. $^{9}$.

As expected, based on the fit of the speed-density models, the linear speed-density model suggested by Greenshields predicting does not fit the real trend of the empirical dataset. According to Greenshields, the capacity occurs at almost half of jam density and half of free flow speed, which does not support the empirical data on the study sections, clearly evident from Figure 8 . Therefore, based on the available dataset at boundary conditions, authors decided to fit single-regime model

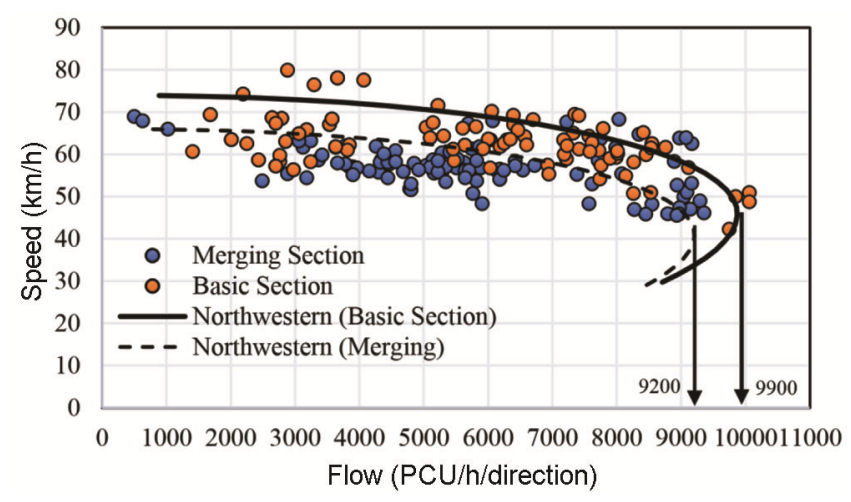

Figure 7. Comparison of speed-flow relationship at merging section and basic section.

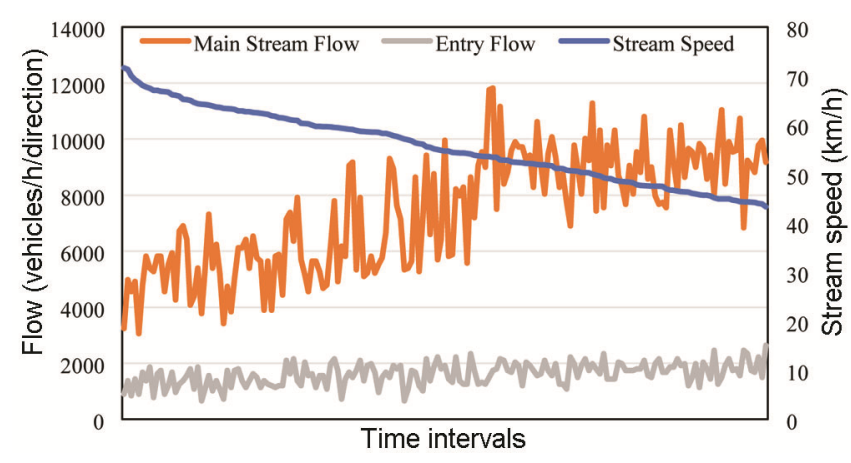

Figure 8. Effect on stream speed with respect to main stream flow and entry flow. proposed by Northwestern University ${ }^{10}$. This model requires the knowledge of free flow speed and optimum density, which can be easily calibrated based on the available empirical dataset. However, the most recent Van Aerde model $^{11}$ could have given better fit since it considers four parameter calibrations in its equation. Due to the unavailability of data in congested conditions, authors decided to choose Northwestern model. The comparison between the relationships showed that there is a significant effect on free speed at merging section when compared with the basic section of the roadway. Around $8 \%$ difference in capacity was observed.

From Figure 7, it is clear that between 9000 and 10,000 $\mathrm{PCU} /$ hour/direction, several data with different flow and same speeds can be observed. The randomness in traffic stream behaviour due to vehicle-to-vehicle interaction at micro-level is the reason that at different flow levels, similar speeds can be maintained. The same is true when the presence of motorized two-wheeler and three-wheeler in the traffic stream is high. The behaviour of justmentioned vehicle types is a bit aggressive under heterogeneous traffic conditions, because of their ability to manoeuvre under non-lane-based movement, even at capacity flow conditions, which makes it possible for the traffic stream to maintain the same speed at higher flow.

Observation of the dataset of merging section shows that speeds are always on the lower side at different flow conditions. The reason for this may be attributed to the fact that speeds are not only influenced by mainstream flow but also due to the entry flow. With this motivation, in the present study, a model was developed which can predict stream speed based on mainstream flow and entry flow. The subsequent section shows the model.

\section{Effect of entry flow on stream speed}

The type of merging phenomena shown in the present study is quite common on high-speed urban roads in India. The stream speed on such type of sections is found to be the function of both flow levels as well as entry flow. To understand the variation more appropriately, stream speed is kept in decreasing order and the mainstream flow and entry flow are also plotted for the same on a three axes plot (Figure 8). It can be easily understood from the figure that with increase in both main

Table 3. Results from regression analysis. Summary of output on $R$-square values

\begin{tabular}{lc}
\hline Multiple $R$ & 0.789009722 \\
$R$ square & 0.622536342 \\
Adjusted $R$ square & 0.61814723 \\
Standard error & 4.476716156 \\
Observations & 175 \\
\hline
\end{tabular}


Table 4. Results from regression analysis. Summary of output on significance of variables

\begin{tabular}{llccc}
\hline & Coefficients & Standard error & $t$ Stat & $P$-value \\
\hline Intercept & 78.66150801 & 1.575854473 & 49.91673366 & $2.8964 \mathrm{E}-104$ \\
Main stream flow & -0.002495525 & 0.00018488 & -13.49811067 & $8.82565 \mathrm{E}-29$ \\
Entry flow & -0.002856349 & 0.000953395 & -2.995976849 & 0.003140095 \\
\hline
\end{tabular}

stream flow and entry flow, there is a substantial decrease in the overall stream speed.

A multi-linear regression model is developed in the present study, where stream speed is kept as dependent variable and the mainstream flow and entry flows are kept as independent variables. The correlation coefficient between mainstream flow and entry flow was found to be 0.44 , which shows variables are not interdependent and can be used as independent variables for the regression analysis. The resultant multi-linear regression model for the relationship is

$$
\begin{aligned}
\text { Stream speed }= & 78.6-0.0025 \times \text { main stream flow } \\
& -0.0028 \times \text { entry flow }
\end{aligned}
$$

The summary output of regression model obtained using Microsoft Excel is presented in Table 3. The result from Table 3 shows slightly lower $R^{2}$ value, which is due to a lesser number of samples. However, an increase in data samples may further increase the $R^{2}$ value. Moreover, all the variables in eq. (1) are found to be statistically significant based on $p$-value given in Table 4. This further proves the significance of the above equation.

\section{Discussion and conclusion}

The research evaluates traffic flow characteristics at the merging section on multi-lane urban roads in Delhi. It may be considered as a representative merging section since similar roadway characteristics are observed at the location of merging over the total length of $28 \mathrm{~km}$ of the roadway under consideration in this study. Also, on multilane highways in India, similar merging sections are provided. In general, there are fewer studies attempted at merging sections on high-speed urban roads in India. Therefore, this study was conducted to study traffic flow characteristics at the location of merging on an eight-lane divided urban road in Delhi. Various traffic macroscopic as well as microscopic traffic flow parameters such as flow, speed and headway are studied at the location of merging to derive preliminary yet very significant findings. Some of the key findings from this study are given below:

- Median side lane (lane 1) and adjacent to median side lane (lane 2) have the maximum percentage of vehicles as cars. However, adjacent to kerbside lane (lane 3) and kerbside lane (lane 4) have composition from all vehicle categories.

- Free speed distribution conducted at merging section and the basic section on same roadway facility shows a reduction in speed of different vehicle categories by $4-14 \%$ at merging section.

- Speed data for individual lanes are found to significantly decrease from median side lanes to kerbside lanes, showing the effect of merging on the speed of vehicles closer to merging location. However, lane 2 is most with very high variation in speeds for heavy flow conditions.

- Generalized extreme value (GEV) distribution is found to be best-fitting to the headway data. The headway study conducted at upstream of merging sections at merging and basic section on the same roadway provides some of the important findings. Under low and moderate flow conditions, vehicles are likely to shift towards lanes which are adjacent to median side lanes to avoid competing for the same space. However, under heavy flow conditions, vehicles are bound to utilize all the lanes. However, at the location of merging, kerbside lanes are also utilized effectively considering merging manoeuver through kerbside lanes.

- Comparison of speed-flow relationship at mid-block and merging section showed that a total reduction in capacity was observed to be about $8 \%$.

- Stream speed is found to be a function of the entry flow and mainstream flow at the location of merging.

The merging section is a potential bottleneck location on high-speed roads and a source of traffic crashes due to the competition of two traffic flows, its operations directly affect the whole efficiency and safety level of the highspeed road networks. Thus, the operations of merging section are being paid much attention for both researchers and practitioners' point of view. The results obtained from this study could be useful in an operational analysis of merging section on the eight-lane divided section with a similar type of merging manoeuver prevailing on various other eight-lane divided roads in Indian conditions. Also, the authors foresee that, it would be helpful in the development of a simulation model for merging section on multilane road. Level of service and safety aspects at the location of merging sections are being studied with the developed simulation model under varying traffic conditions using some of the outputs from the present study. 


\section{RESEARCH ARTICLES}

Moreover, the present findings would also be useful for identification of the optimum road geometrics, which can improve efficiency and safety at the location of merging. These findings will be used in the development of a simulation model for similar sections. Further, some changes can be made in the road geometrics and its after-effects can be checked in the simulation model. The iterative procedure can be useful in finalizing the most adequate section at the location of merging. The study can also be extended towards designing an appropriate length of acceleration lane at the location of merging.

1. Elefteriadou, L., Roess, R. P. and McShane, W. R., Probabilistic nature of breakdown at freeway merge junctions. Trans. Res. Rec., 1995, 1484(1484), 80-89.

2. Kerner, B. and Rehborn, H., Experimental properties of complexity in traffic flow. Phys. Rev. E, 1996, 53(5), R4275-R4278; https://doi.org/10.1103/PhysRevE.53.R4275.

3. Kerner, B. S. and Rehborn, H., Experimental properties of phase transitions in traffic flow. Phys. Rev. Lett., 1997, 79, 4030-4033; https://doi.org/10.1103/PhysRevLett.79.4030.

4. Kondyli, A. and Elefteriadou, L., Modeling driver behavior at freeway-ramp merges. Transp. Res. Rec.: J. Trans. Res. Board, 2011, 2249(2249), 29-37; https://doi.org/10.3141/2249-05.

5. Sarvi, M. and Kuwahara, M., Microsimulation of freeway ramp merging processes under congested traffic conditions. IEEE Trans.
Intel. Trans. Syst., 2007, 8(3), 470-479; https://doi.org/10.1109/ TITS.2007.895305.

6. Sarvi, M. and Kuwahara, M., Using ITS to improve the capacity of freeway merging sections by transferring freight vehicles. IEEE Trans. Intell. Transp. Syst., 2008, 9(4), 580-588; https://doi.org/ 10.1109/TITS.2008.2006812.

7. Weng, J., Xue, S. and Yan, X., Modeling vehicle merging behavior in work zone merging areas during the merging implementation period. IEEE Trans. Intell. Trans. Syst., 2016, 17(4), 917925; https://doi.org/10.1109/TITS.2015.2477335.

8. AASHTO, A policy on geometric design of highways and streets. Washington, DC, 2011.

9. Kumar, P. Arkatkar, S., Joshi, G. and Dhamaniya, A., New Methodology for Estimating PCU On Multi Lane Urban Roads Under Mixed Traffic Scenario Based on Area Occupancy 96th Annual TRB meeting Proceedings, Washington, DC, 2017.

10. Drake, J. S., Schofer, J. L. and May Jr, A. D., A statistical analysis of speed-density hypotheses. Highway Res. Rec., 1967, 154, 112117.

11. Rakha, H. A., Validation of Van Aerde's simplified steady-state car-following and traffic stream model. Trans. Lett.: Int. J. Transp. Res., 2009, 1(3), 227-244; https://doi.org/10.3328/Tl. 2009.01.03.227-244.

Received 18 October 2017; revised accepted 5 March 2019

doi: $10.18520 / \mathrm{cs} / \mathrm{v} 117 / \mathrm{i} 1 / 94-103$ 